\title{
Acumulación, removilización, absorción postantesis y eficiencia de utilización de nitrógeno en trigo bajo diferentes labranzas y fertilizaciones ${ }^{(1)}$
}

\author{
Silvina Inés Golik(2), Hugo Oscar Chidichimo(2), Diego Pérez ${ }^{(2)}$ y Luciano Pane ${ }^{(2)}$
}

Resumen - El objetivo de este trabajo fue analizar la acumulación, removilización, absorción postantesis y la eficiencia en la utilización de $\mathrm{N}$ bajo dos sistemas de labranza (reja y cincel) y diferentes tipos de fertilización, en los cultivares de trigo pan Buck Pronto y Buck Charrúa. Los tratamientos de fertilización fueron: testigo sin el agregado de $\mathrm{N}$, aplicación de $90 \mathrm{~kg} \mathrm{ha}^{-1} \mathrm{de} \mathrm{N}$ como urea a la siembra, aplicación de $90 \mathrm{~kg} \mathrm{ha}^{-1} \mathrm{de} \mathrm{N}$ como nitrato de amonio calcáreo a la siembra y aplicación de $\mathrm{N}$ como urea y como nitrato de amonio calcáreo en dosis de $45 \mathrm{~kg} \mathrm{ha}^{-1}$ a la siembra más $45 \mathrm{~kg} \mathrm{ha}^{-1}$ a fin de macollaje, respectivamente. Se encontraron diferencias en el $\mathrm{N}$ acumulado entre sistemas de labranzas; con cincel se acumuló $18 \mathrm{~kg} \mathrm{ha}^{-1}$ más que bajo reja, pero esto no se tradujo en el porcentaje de $\mathrm{N}$ contribuido al grano por removilización ni por absorción postantesis. La fertilización aumentó el $\mathrm{N}$ acumulado en un promedio de $50 \mathrm{~kg} \mathrm{ha}^{-1}$. A su vez provocó un aumento en la contribución al grano del $\mathrm{N}$ removilizado, pero no mejoró el aporte del $\mathrm{N}$ absorbido postantesis, ni aún en la aplicación en dos momentos. Los cultivares presentaron diferencias en el $\mathrm{N}$ acumulado y en el porcentaje aportado al grano por removilización y absorción postantesis. La eficiencia de utilización del $\mathrm{N}$ dependió de la labranza y de la fertilización.

Términos para índice: Triticum aestivum, reja y vertedera, cincel, fertilización, cultivares.

\section{Nitrogen accumulation, translocation, postanthesis uptake and utilization efficiency under different tillage systems and fertlizer types}

\begin{abstract}
The objective of this work was to analyse $\mathrm{N}$ accumulation, translocation, postanthesis uptake and utilization efficiency under two tillage systems (moldboard plow and chisel plow) and different fertilizer types in two cultivars of bread wheat (Buck Pronto and Buck Charrúa). The N fertilizer treatments were: without $\mathrm{N}, 90 \mathrm{~kg} \mathrm{ha}^{-1}$ at sowing as urea, $90 \mathrm{~kg} \mathrm{ha}^{-1}$ at sowing as ammonium nitrate, $45 \mathrm{~kg} \mathrm{ha}^{-1}$ at sowing and $45 \mathrm{~kg} \mathrm{ha}^{-1}$ at tillering as urea and $45 \mathrm{~kg} \mathrm{ha}^{-1}$ at sowing and $45 \mathrm{~kg} \mathrm{ha}^{-1}$ at tillering as ammonium nitrate. Difference in $\mathrm{N}$ accumulation between tillage systems (chisel accumulated $18 \mathrm{~kg} \mathrm{ha}^{-1}$ more than moldboard) was found, but this did not correspond to improvement of grain $\mathrm{N}$ content by remobilization or by postanthesis $\mathrm{N}$ uptake. Fertilization, independently of the type and time of application, increased $\mathrm{N}$ accumulated (50 kg ha-1 in average) and $\mathrm{N}$ remobilized to the grain, but $\mathrm{N}$ contribution to grain arising from postanthesis uptake did not improve. Cultivars presented differences in $\mathrm{N}$ accumulated and in $\mathrm{N}$ contribution to grain by remobilization and postanthesis uptake. Nitrogen utilization efficiency depended on the tillage systems and the fertilization treatments.
\end{abstract}

Index terms: Triticum aestivum, mouldboard ploughs, chisel ploughs, fertilization, cultivars.

\section{Introducción}

El $\mathrm{N}$ es un elemento esencial para lograr altos rendimientos y calidad en trigo. Su absorción y partición se encuentra determinada en gran medida

(1) Aceptado para publicación en 4 de febrero de 2003 .

(2) Universidad Nacional de La Plata, Facultad de Ciencias Agrarias y Forestales, Departamento de Tecnología Agropecuaria y Forestal, Cerealicultura, CC 31 (1900), La Plata, Argentina. E-mail: cereali@ceres.agro.unlp.edu.ar por la disponibilidad y demanda durante varios estados fenológicos del cultivo (Campbell \& De Jong, 2000). Se conoce que el $\mathrm{N}$ absorbido previo a la antesis, en los cereales invernales, representa alrededor del $50 \%$ al $75 \%$ del $\mathrm{N}$ total en la planta a cosecha (Austin et al., 1976; Spiertz \& Ellen, 1978; Heitholt et al., 1990), dependiendo entre otros factores del cultivar empleado (Takahashi et al., 1996). En condiciones de alta fertilidad del suelo la absorción postantesis resulta igualmente importante porque 
está positivamente correlacionada con la proteína del grano y con el índice de cosecha de N (Spiertz \& Ellen, 1978; Perez et al., 1983; Sarandón \& Caldiz, 1990; Sarandón et al., 1997). Esta absorción se encuentra mucho más influenciada por los factores ambientales como la temperatura, humedad y disponibilidad de agua y N (Papakosta \& Gagianas, 1991) que la absorción previa a la antesis. La reducción en los niveles de absorción postantesis es común en situaciones normales a campo donde la disponibilidad de $\mathrm{N}$ a partir de floración se hace escasa. Si la restricción que impone el agotamiento del $\mathrm{N}$ del suelo se supera con ofertas mayores (por ejemplo con aportes por fertilización en dosis fraccionadas) se pueden observar aumentos en la absorción postfloración (Osaki et al., 1997; Delogu et al., 1998; Ruiz, 2001). En este caso la magnitud del $\mathrm{N}$ absorbida hasta floración también es mayor, pero la proporción que representa del total absorbido hasta la madurez se reduce (Spiertz \& Ellen, 1978; Campbell et al., 1983; Dalling, 1985; Delogu et al., 1998; Ruiz, 2001). En la medida que la restricción nitrogenada es menos marcada, la curva de acumulación sigue la marcha de la acumulación de la materia seca (Ruiz, 2001). Palta \& Fillery (1993) indicaron que frente a dosis creciente de $\mathrm{N}$ aplicado a la siembra, se incrementó el $\mathrm{N}$ en el grano proveniente del $\mathrm{N}$ acumulado previo a la antesis y disminuyó el proveniente de la absorción postantesis.

El tipo de labranza también puede modificar la dinámica del $\mathrm{N}$, incidiendo en la acumulación y la eficiencia en la utilización del mismo por el cultivo (Sarandón et al., 1997). En general, se considera que la disponibilidad de $\mathrm{N}$ es más rápida bajo labranza convencional que en siembra directa, aunque la cantidad de $\mathrm{N}$ mineralizado puede ser suficiente para el desarrollo del cultivo en ambos sistemas (Doran, 1980). La labranza con cinceles puede mostrar un comportamiento intermedio entre reja y siembra directa, respecto de la dinámica de dicho nutriente (Alvarez et al., 1998), atenuando el problema de la insuficiente disponibilidad de $\mathrm{N}$ y la menor respuesta a la fertilización encontrada en siembra directa, frecuentemente, asociada a una mayor compactación (Balbuena et al., 1996; Ferreras et al., 2001) o al menos a un endurecimiento superficial del suelo (Taboada et al., 1998).
Tanto el momento de fertilización, como el sistema de labranza tienen efectos importantes en la recuperación del N por el cultivo (Golik \& Chidichimo, 2001, 2002; Sarquis et al., 2002), aunque la eficiencia del uso del $\mathrm{N}$ también puede variar según el tipo de fertilizante utilizado. La urea es el fertilizante más usado, aunque en sistemas con residuos en superficie puede llevar a pérdidas importantes por volatilización y su aprovechamiento se retrasa hasta que se ha hidrolizado en el suelo. La utilización de fertilizantes compuestos, como el nitrato de amonio podría mejorar la eficiencia con respecto a la urea, tal como lo sugieren Peruzzo et al. (1994).

En Argentina el trigo se cultiva tradicionalmente con un área promedio de 5 millones de hectáreas. En los últimos años, la degradación de los suelos producida por la intensificación de la agricultura, principalmente en la Pampa Ondulada, y el aumento en la importancia del contenido proteico del grano a partir de su inclusión en el estándar de comercialización, requieren compatibilizar el uso de técnicas conservacionistas del suelo con un adecuado suministro de $\mathrm{N}$ al cultivo.

El objetivo de este trabajo fue analizar la acumulación, removilización, absorción postantesis y la eficiencia en la utilización del $\mathrm{N}$ bajo diferentes sistemas de labranza, momentos de suministro y tipos de fertilizante.

\section{Material y Métodos}

Durante 1997 se realizó un ensayo en la Estación Experimental J.J. Hirschhorn (35\% LS), Facultad de Ciencias Agrarias y Forestales, La Plata, Argentina, sobre un Argiudol Típico con las siguientes características a la siembra $(0-20 \mathrm{~cm})$ : MO, 47,9 $\mathrm{g} \mathrm{kg}^{-1} ; \mathrm{N}$ total, $2,5 \mathrm{~g} \mathrm{~kg}^{-1}$; $\mathrm{C}$ org, 27,8 $\mathrm{g} \mathrm{kg}^{-1}$; relación C/N, 11,2; P asimilable (Bray Kurtz), $7 \mathrm{mg} \mathrm{kg}^{-1}$ y cuya historia previa fue: trigo, maíz, soja, maíz; bajo labranza convencional. El 27 de julio se sembraron los cultivares de trigo pan Buck Pronto (BP) y Buck Charrúa (BCh), bajo los sistemas de labranza con arado de reja y vertedera (reja) y labranza con cincel (cincel) en parcelas de $3,8 \times 12 \mathrm{~m}$ de largo, a una densidad de 350 plantas $\mathrm{m}^{-2}$. En cada sistema de labranza se aplicaron los siguientes tratamientos de fertilización: testigo sin $\mathrm{N}$ (N0), $90 \mathrm{~kg} \mathrm{ha}^{-1}$ de $\mathrm{N}$ como urea a la siembra (U90), $90 \mathrm{~kg} \mathrm{ha}^{-1}$ de $\mathrm{N}$ como nitrato de amonio calcáreo a la siem bra (Ni90), $\mathrm{N}$ aplicado como urea en dosis de $45 \mathrm{~kg} \mathrm{ha}^{-1}$ a la siembra más $45 \mathrm{~kg} \mathrm{ha}^{-1}$ a fin de macollaje (U45+45) y N 
aplicado como nitrato de amonio calcáreo en dosis de $45 \mathrm{~kg} \mathrm{ha}^{-1}$ a la siembra más $45 \mathrm{~kg} \mathrm{ha}^{-1}$ a fin de macollaje (Ni45+45). A todas las parcelas se les aplicó $100 \mathrm{~kg} \mathrm{ha}^{-1}$ de superfosfato triple en el momento de la siembra. El ensayo quedó formado por dos tratamientos de labranza, dos cultivares y cinco tratamientos de fertilización, según un diseño factorial $2 \times 2 \times 5$ en bloques al azar con tres repeticiones.

A fin de macollaje (2 de octubre), antesis ( 29 de octubre) y madurez (24 de diciembre), estados de crecimiento 29 , 65 y 91 de Zadoks et al. (1974), respectivamente, se obtuvo la biomasa a partir de la extracción de una superficie de $0,3 \mathrm{~m}^{2}$ (sectores de $0,5 \mathrm{~m}$ de tres surcos contiguos) y su posterior secado en estufa a $70^{\circ} \mathrm{C}$ durante 48 horas .

Se determinó el porcentaje de N por micro-Kjeldahl, para las distintas etapas fenológicas consideradas previa división del material en tallo y espiga en antesis y en paja, granza y granos en madurez. Fueron determinados el $\mathrm{N}$ acumulado $(\mathrm{N})$ en $\mathrm{kg} \mathrm{ha}^{-1}$, a partir del porcentaje de $\mathrm{N}$ por la materia seca correspondiente; la acumulación de $\mathrm{N}$ postantesis (absorbido del suelo durante el llenado de grano) $\left(\mathrm{kg} \mathrm{ha}^{-1}\right)$ como la diferencia entre el contenido de $\mathrm{N}$ en la planta a madurez y en antesis; la contribución al grano del $\mathrm{N}$ absorbido o acumulado postantesis: obtenido por la relación entre el $\mathrm{N}$ acumulado postantesis y el $\mathrm{N}$ contenido en el grano a madurez; la contribución al grano del $\mathrm{N}$ removilizado desde los órganos vegetativos durante el llenado de grano calculada por la relación entre la disminución del $\mathrm{N}$ en los órganos vegetativos desde antesis hasta madurez y el $\mathrm{N}$ contenido en el grano a madurez; el índice de cosecha del $\mathrm{N}$ como la relación entre el $\mathrm{N}$ en el grano y el $\mathrm{N}$ de la planta entera a madurez; la eficiencia en la utilización del $\mathrm{N}$ en $\mathrm{kg} \mathrm{kg}^{-1}$ como la relación entre el rendimiento y el $\mathrm{N}$ acumulado a madurez; la eficiencia agronómica en kg kg-1 como la relación: (rendim con N90 rendim con N0)/kg de N aplicado en N90; la eficiencia fisiológica como la relación: (rendim con N90 - rendim con N0)/ ( $\mathrm{N}$ acumulado con N90 - N acumulado con N0); la fracción aparente recuperada (\%) como la relación: ( $\mathrm{N}$ acumulado con N90 - N acumulado con N0)/ $\mathrm{N}$ aplicado en N90.

Los resultados se procesaron con un análisis de varianza y las medias se compararon según la prueba de Tukey al 0,05 de probabilidades. Además se analizaron las posibles correlaciones entre las variables planteadas.

\section{Resultados y Discusión}

La acumulación de $\mathrm{N}$ y la contribución al grano, del $\mathrm{N}$ removido de los tejidos vegetativos y del absorbido postantesis, fueron afectados en forma diferente por los sistemas de labranza, los tratamientos de fertilización y los cultivares en las etapas fenológicas consideradas (Cuadros 1 y 2).

Las condiciones ambientales que prevalecieron durante el ensayo permitieron buenos rendimientos aún en las parcelas testigo, ya que si bien las precipitaciones fueron escasas durante el mes de septiembre, tanto en los meses previos como en los posteriores resultaron elevadas, comparadas con las medias mensuales históricas, atendiendo los requerimientos del cultivo (Figura 1). En macollaje la acumulación de $\mathrm{N}$ no difirió entre los sistemas de labranza, a pesar de que la menor disponibilidad temprana de $\mathrm{N}$ para el cultivo en los sistemas de labranza conservacionista (Doran, 1980) debería

Cuadro 1. Nitrógeno acumulado $\left(\mathrm{kg} \mathrm{ha}^{-1}\right)$ en macollaje, antesis y madurez en dos cultivares de trigo pan bajo diferentes labranzas y tratamientos de fertilización nitrogenada ${ }^{(1)}$.

\begin{tabular}{|c|c|c|c|c|c|c|c|}
\hline \multirow[t]{2}{*}{ Tratamiento $^{(2)}$} & \multirow{2}{*}{$\begin{array}{c}\text { Macollaje } \\
\text { Planta } \\
\end{array}$} & \multicolumn{3}{|c|}{ Antesis } & \multicolumn{3}{|c|}{ Madurez } \\
\hline & & Espiga & Tallo & Planta & Paja+granza & Grano & Planta \\
\hline \multicolumn{8}{|l|}{ Labranza } \\
\hline Reja & $79,8 \mathrm{a}$ & $26,3 b$ & $116,6 a$ & $142,2 b$ & $47,0 \mathrm{~b}$ & $148,7 \mathrm{a}$ & $195,4 b$ \\
\hline Cincel & $77,6 a$ & $34,1 \mathrm{a}$ & $129,8 \mathrm{a}$ & $160,0 \mathrm{a}$ & $59,0 \mathrm{a}$ & $157,2 \mathrm{a}$ & $216,9 a$ \\
\hline \multicolumn{8}{|l|}{ Fertilización } \\
\hline N0 & $53,3 \mathrm{c}$ & $18,2 b$ & $71,1 \mathrm{~b}$ & $88,0 \mathrm{~b}$ & $37,0 \mathrm{c}$ & $124,4 b$ & $161,5 b$ \\
\hline U90 & $94,6 a$ & $32,0 \mathrm{a}$ & $132,0 \mathrm{a}$ & $162,3 \mathrm{a}$ & $59,5 \mathrm{ba}$ & $165,6 a$ & $225,1 \mathrm{a}$ \\
\hline $\mathrm{Ni} 90$ & $96,1 \mathrm{a}$ & $35,5 \mathrm{a}$ & $143,5 \mathrm{a}$ & $173,0 \mathrm{a}$ & $51,1 \mathrm{~b}$ & $156,8 \mathrm{a}$ & $208,0 \mathrm{a}$ \\
\hline $\mathrm{U} 45+45$ & $78,0 \mathrm{~b}$ & $32,4 \mathrm{a}$ & $133,9 \mathrm{a}$ & $166,2 \mathrm{a}$ & $63,1 \mathrm{a}$ & $157,3 a$ & $220,4 a$ \\
\hline $\mathrm{Ni} 45+45$ & $71,5 b$ & $33,0 \mathrm{a}$ & $135,5 \mathrm{a}$ & $166,1 \mathrm{a}$ & $55,1 \mathrm{ba}$ & $160,8 \mathrm{a}$ & $215,9 \mathrm{a}$ \\
\hline \multicolumn{8}{|l|}{ Cultivares } \\
\hline Buck Pronto & $76,8 \mathrm{a}$ & $33,6 a$ & $118,4 \mathrm{a}$ & $152,0 \mathrm{a}$ & $52,3 \mathrm{a}$ & $177,3 \mathrm{a}$ & $229,6 a$ \\
\hline Buck Charrúa & $80,6 \mathrm{a}$ & $26,8 b$ & $128,0 \mathrm{a}$ & $150,2 \mathrm{a}$ & $54,0 \mathrm{a}$ & $128,7 \mathrm{~b}$ & $182,7 \mathrm{~b}$ \\
\hline
\end{tabular}

${ }^{(1)}$ Letras distintas en la misma columna indican diferencias estadísticamente significativas entre tratamientos $(\mathrm{P}=0,05)$ por el test de Tukey.(2)Reja: arado de reja y vertedera; Cincel: arado de cinceles; N0: testigo sin aplicación de N; U90: aplicación de $90 \mathrm{~kg} \mathrm{ha}^{-1}$ de N como urea a la siembra; Ni90: aplicación de $90 \mathrm{~kg} \mathrm{ha}^{-1}$ de $\mathrm{N}$ como nitrato de amonio calcáreo a la siembra; U45+45: $\mathrm{N}$ aplicado como urea en dosis de $45 \mathrm{~kg}$ ha ${ }^{-1}$ a la siembra más $45 \mathrm{~kg} \mathrm{ha}^{-1}$ a fin de macollaje; Ni45+45: N aplicado como nitrato de amonio calcáreo en dosis de $45 \mathrm{~kg} \mathrm{ha}^{-1}$ a la siembra más $45 \mathrm{~kg}^{-1} \mathrm{a}^{-1}$ a fin de macollaje 
traducirse en una mayor respuesta a la aplicación de fertilizantes (no hubo interacciones entre las labranzas y los tratamientos de fertilización).

Cuadro 2. Nitrógeno acumulado postantesis, porcentaje de $\mathrm{N}$ en el grano proveniente del removilizado y del absorbido postantesis en dos cultivares de trigo pan bajo diferentes labranzas y tratamientos de fertilización nitrogenada ${ }^{(1)}$.

\begin{tabular}{lccc}
\hline Tratamiento $^{(2)}$ & $\begin{array}{c}\mathrm{N} \text { postantesis } \\
\left(\mathrm{kg} \mathrm{ha}^{-1}\right)\end{array}$ & $\begin{array}{c}\mathrm{N} \text { removilizado } \\
(\%)\end{array}$ & $\begin{array}{c}\mathrm{N} \text { absorbido llenado } \\
\text { de grano } \\
(\%)\end{array}$ \\
\hline $\begin{array}{l}\text { Labranza } \\
\text { Reja }\end{array}$ & $53,3 \mathrm{a}$ & $47,8 \mathrm{a}$ & $34,7 \mathrm{a}$ \\
Cincel & $56,8 \mathrm{a}$ & $48,6 \mathrm{a}$ & $31,6 \mathrm{a}$ \\
\hline Fertilización & & & \\
N0 & $73,5 \mathrm{a}$ & $28,8 \mathrm{~b}$ & $57,6 \mathrm{a}$ \\
U90 & $62,8 \mathrm{ab}$ & $47,6 \mathrm{a}$ & $34,3 \mathrm{~b}$ \\
Ni90 & $34,9 \mathrm{~b}$ & $62,1 \mathrm{a}$ & $18,6 \mathrm{~b}$ \\
U45+45 & $54,3 \mathrm{ab}$ & $47,2 \mathrm{a}$ & $31,3 \mathrm{~b}$ \\
Ni45+45 & $49,8 \mathrm{ab}$ & $55,2 \mathrm{a}$ & $24,0 \mathrm{~b}$ \\
\hline Cultivares & & & \\
Buck Pronto & $77,69 \mathrm{a}$ & $39,32 \mathrm{~b}$ & $41,10 \mathrm{a}$ \\
Buck Charrúa & $32,44 \mathrm{~b}$ & $57,05 \mathrm{a}$ & $25,25 \mathrm{~b}$ \\
\hline
\end{tabular}

(1)Letras distintas en la misma columna indican diferencias estadísticamente significativas entre tratamientos $(\mathrm{P}=0,05)$ por el test de Tukey. ${ }^{(2)}$ Reja: arado de reja y vertedera; Cincel: arado de cinceles; N0: testigo sin aplicación de N; U90: aplicación de $90 \mathrm{~kg} \mathrm{ha}^{-1}$ de $\mathrm{N}$ como urea a la siembra; Ni90: aplicación de $90 \mathrm{~kg} \mathrm{ha}^{-1}$ de $\mathrm{N}$ como nitrato de amonio calcáreo a la siembra; U45+45: $\mathrm{N}$ aplicado como urea en dosis de $45 \mathrm{~kg} \mathrm{ha}^{-1}$ a la siembra más $45 \mathrm{~kg} \mathrm{ha}^{-1}$ a fin de macollaje; Ni45+45: $\mathrm{N}$ aplicado como nitrato de amonio calcáreo en dosis de $45 \mathrm{~kg} \mathrm{ha}^{-1}$ a la siembra más $45 \mathrm{~kg} \mathrm{ha}^{-1}$ a fin de macollaje.
Se observó un efecto significativo de la fertilización sobre la acumulación de $\mathrm{N}$, independientemente del tipo de fertilizante utilizado. A su vez se encontraron diferencias entre la aplicación de $\mathrm{N}$ en una sola dosis y la aplicación dividida, cuando hubo mayor disponibilidad inicial la acumulación fue mayor. Los cultivares no presentaron diferencias en la acumulación de $\mathrm{N}$ en esta etapa.

En antesis el $\mathrm{N}$ acumulado fue mayor en cincel que en reja; se incrementó con el agregado de $\mathrm{N}$, independientemente del momento de aplicación y del tipo de fertilizante y no difirió entre cultivares. Considerando los distintos componentes de la planta, la acumulación de $\mathrm{N}$ en la espiga resultó afectada por todos los tratamientos; no así su acumulación en el tallo que sólo presentó diferencias significativas para los tratamientos de fertilización.

El N acumulado a madurez también fue mayor para el cincel, aumentó con la fertilización y presentó diferencias entre cultivares, mostrando Buck Pronto mayor respuesta. Tanto el $\mathrm{N}$ acumulado en grano como el acumulado en paja+granza presentaron una respuesta similar a la obtenida en el estado fenológico anteriormente considerado.

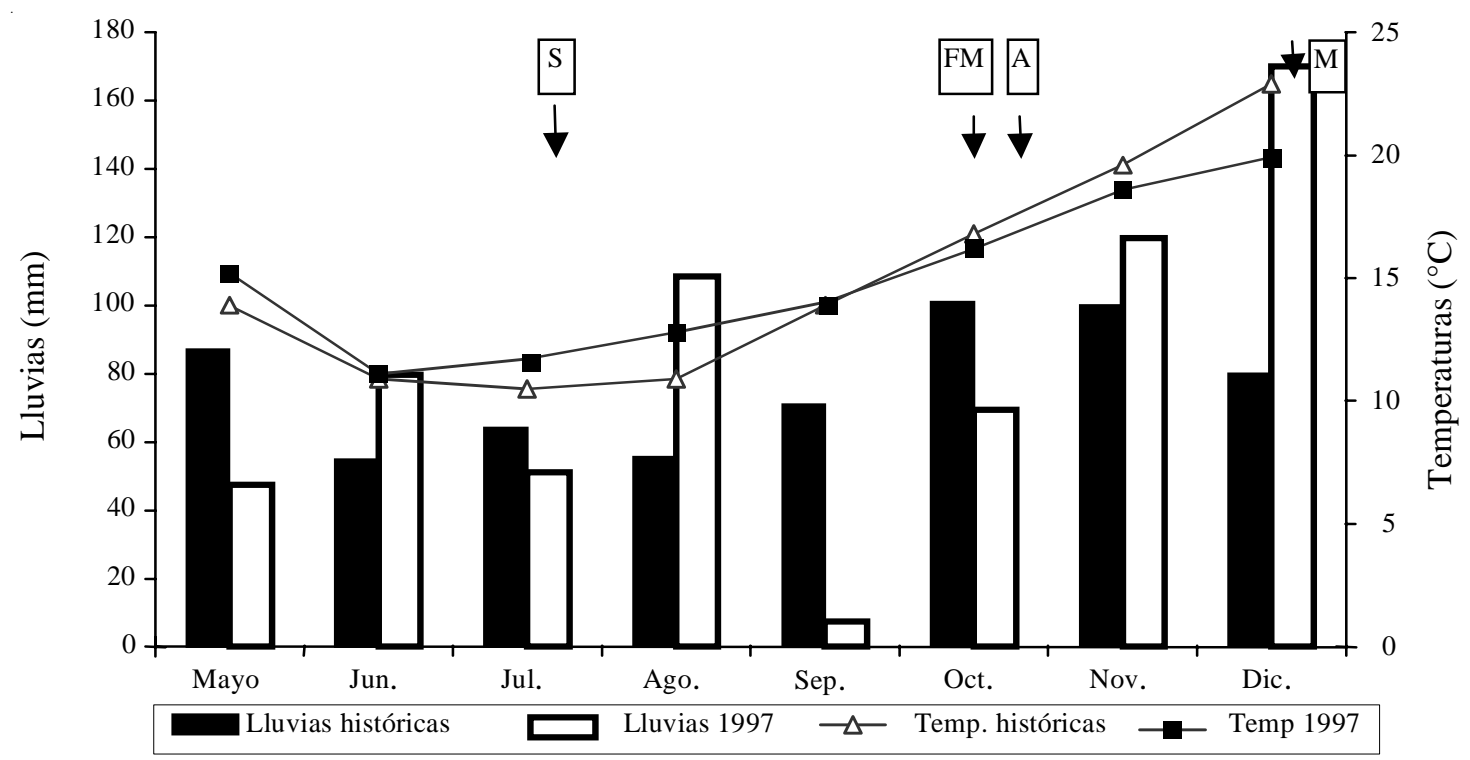

Figura 1. Precipitaciones y temperaturas medias mensuales históricas y durante el desarrollo del cultivo de trigo (S: siembra, 27 de julio; FM: fin de macollaje, 2 de octubre; A: antesis, 29 de octubre; M: madurez, 24 de diciembre). La Plata, Argentina. 
A pesar de lo sugerido por Peruzzo et al. (1994), no hubo una respuesta diferencial en el $\mathrm{N}$ acumulado en esta etapa, como en ninguna de las anteriores, entre los tratamientos fertilizados con urea y los fertilizados con nitrato de amonio (Cuadro 1). La cantidad de $\mathrm{N}$ acumulado en el cultivo estuvo más relacionada con la biomasa $(r=0,92 ; n=50)$, que con la concentración del $\mathrm{N}$ en la planta $(\mathrm{r}=0,42$; $\mathrm{n}=50$ ), tal como ya se había encontrado por Sarandón et al. (1997). No obstante hacia el final del ciclo del cultivo, durante el llenado de grano, su tasa de acumulación fue menor que la de la biomasa, lo que podría estar indicando una falta de disponibilidad de dicho nutriente según lo sugerido por Ruiz (2001) (Figura 2). Ambas variables aumentaron significativamente con el agregado de $\mathrm{N}$, independientemente del tipo de fertilizante. La biomasa para el tratamiento testigo y para los fertilizados (promedio), fue en macollaje de $2.320 \mathrm{~kg} \mathrm{ha}^{-1}$ y $3.270 \mathrm{~kg} \mathrm{ha}^{-1}$, en antesis $6.630 \mathrm{~kg} \mathrm{ha}^{-1}$ y $9.340 \mathrm{~kg} \mathrm{ha}^{-1}$ y en madurez de $13.310 \mathrm{~kg} \mathrm{ha}^{-1}$ y $15.420 \mathrm{~kg} \mathrm{ha}^{-1}$, respectivamente.

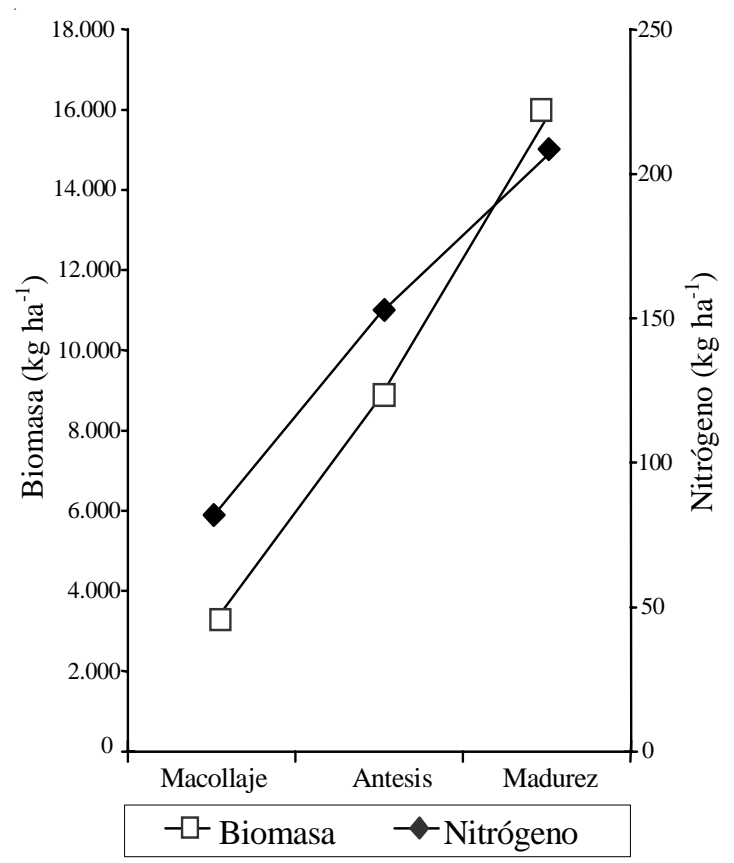

Figura 2. Valores promedios de todos los tratamientos de acumulación de biomasa y de nitrógeno durante el ciclo del cultivo de trigo.
Entre antesis y madurez (80-146 días después de la siembra) el aporte del $\mathrm{N}$ removilizado hacia la espiga fue de $29 \%$ para el testigo y $52 \%$, en promedio, para los tratamientos fertilizados. Esta removilización no difirió entre los sistemas de labranzas, a pesar de la mayor acumulación de $\mathrm{N}$ en la planta bajo cincel y, a su vez, fue mayor para Buck Charrúa que para Buck Pronto.

Normalmente, durante el llenado, existe menor disponibilidad de $\mathrm{N}$ como consecuencia de la gran absorción realizada por el cultivo en estadios previos (principalmente desde macollaje hasta antesis), juntamente, para la región triguera, con variabilidad en la disponibilidad de agua asociada a la mayor incidencia de factores ambientales como son las elevadas temperaturas y la ocurrencia de precipitaciones (Figura 1). La contribución al grano del $\mathrm{N}$ absorbido durante el llenado de grano no presentó diferencias entre los sistemas de labranza y disminuyó o no presentó diferencias en los tratamientos fertilizados respecto al testigo; probablemente esto estuvo asociado a la temprana utilización por el cultivo del $\mathrm{N}$ aplicado, generando mayor número de macollos y por ende mayor biomasa tanto en macollaje como en antesis unido a las condiciones ambientales normales en la zona durante el desarrollo de grano. El porcentaje absorbido por el grano en esta etapa fue mayor para Buck Pronto que para Buck Charrúa, coincidiendo con su mayor acumulación de $\mathrm{N}$ postantesis (Cuadro 2).

Si bien la labranza con cincel presentó mayor acumulación de $\mathrm{N}$ tanto en antesis como en madurez, esto no se tradujo en un mayor aporte al grano, ni del $\mathrm{N}$ removilizado ni del absorbido postantesis.

Respecto a la fertilización, los resultados del presente trabajo coinciden con los encontrados por Palta \& Fillery (1993) y no por lo propuesto por Delogu et al. (1998) que hallaron un incremento en el aporte al grano tanto del $\mathrm{N}$ removilizado como del absorbido postantesis trabajando con aplicaciones de altas dosis de N (140 a $210 \mathrm{~kg} \mathrm{ha}^{-1}$ ). En el presente trabajo la fertilización funcionó como en el trabajo de Palta \& Fillery (1993), ya que la aplicación de $\mathrm{N}$ en todos los tratamientos fertilizados provocó un incremento en la contribución a la espiga solamente del $\mathrm{N}$ removilizado y no de ambos, removilizado y absorbido postantesis. Probablemente esto sea debido a que 
las dosis aplicadas en ambos casos fueron similares y relativamente más baja que las aplicadas por Delogu et al. (1998). Por otro lado, si bien generalmente una aplicación tardía de $\mathrm{N}$ o el mantenimiento de una alta concentración en etapas avanzadas del cultivo se asocia a una mayor absorción postantesis (Calderini et al, 1996); la falta de una respuesta diferencial, en el presente trabajo, entre los tratamientos con el agregado de $\mathrm{N}$ en una sola aplicación inicial y una aplicación dividida muy probablemente estuvo asociada a que la segunda fracción de esta última aplicación al ser agregada en macollaje haya sido absorbida preantesis y por lo tanto formado parte del $\mathrm{N}$ removilizado al grano.

Se encontró diferencia varietal entre el $\mathrm{N}$ acumulado tanto en preantesis como en postantesis y madurez, es decir diferencias en el ritmo de acumulación del nutriente, tal lo encontrado por Tanoni \& Cogliatti (1998); Buck Pronto presentó mayor $\mathrm{N}$ acumulado a madurez, principalmente debido a su mayor absorción postantesis respecto a Buck Charrúa, lo que permitió que su contribución al $\mathrm{N}$ del grano fuese mayor que la del otro cultivar y de acuerdo a las correlaciones halladas (Cuadro 2) eso se correspondió con un mayor porcentaje de $\mathrm{N}$ en grano y mayor $\mathrm{N}$ grano $\left(\mathrm{kg} \mathrm{ha}^{-1}\right)$. Buck Charrúa, si bien hasta antesis no tuvo diferencias significativas en el $\mathrm{N}$ acumulado con Buck Pronto, presentó mayor contribución al grano del $\mathrm{N}$ removilizado que este último.

De las correlaciones analizadas entre el porcentaje $\mathrm{N}$ grano, $\mathrm{N}$ grano $(\mathrm{mg})$ y $\mathrm{N}$ grano $\left(\mathrm{kg} \mathrm{ha}^{-1}\right)$ y las variables de absorción y cosecha del $\mathrm{N}$, el porcentaje de $\mathrm{N}$ mostró correlación con el $\mathrm{N}$ acumulado postantesis y en madurez; el $\mathrm{N}$ grano (mg) sólo tuvo correlación con el $\mathrm{N}$ acumulado en madurez y el índice de cosecha de $\mathrm{N}$ y el $\mathrm{N}$ grano $\left(\mathrm{kg} \mathrm{ha}^{-1}\right)$ presentó correlación con todas las variables (Cuadro 3). Esto coincide en gran medida con la hallado por Tanoni \& Cogliatti (1998).

La eficiencia de utilización del $\mathrm{N}$ disminuyó con el agregado de $\mathrm{N}$, para ambos tipos de fertilizantes y momentos de aplicación. Mientras que la eficiencia de utilización del $\mathrm{N}$ fue de $33 \mathrm{~kg} \mathrm{~kg}^{-1}$ en el tratamiento testigo, la misma disminuyó a (promedio de los tratamientos fertilizados) $29 \mathrm{~kg} \mathrm{~kg}^{-1}$ en $\operatorname{los}$ tratamientos fertilizados. La eficiencia de utilización del $\mathrm{N}$ refleja la habilidad de la planta en transformar el $\mathrm{N}$ absorbido en rendimiento económico (grano). Este parámetro es frecuentemente utilizado para comparar diferentes especies o cultivares a con distintos niveles de fertilidad. Delogu et al. (1998) encontraron que la cebada es más eficiente en la utilización del $\mathrm{N}$ que el trigo y esto a su vez se relaciona con el índice de cosecha del $\mathrm{N}$, y que ambos parámetros disminuyen a medida que se incrementa la dosis del fertilizante. En el presente trabajo eficiencia de utilización del $\mathrm{N}$ y el índice de cosecha del $\mathrm{N}$ fueron modificados por el sistema de labranza. Si bien el N acumulado a madurez fue mayor en cincel que en reja, la eficiencia de utilización y de partición del mismo hacia el grano fue inversa. Asimismo, la eficiencia de utilización del $\mathrm{N}$ resultó mayor cuando no se aplicó $\mathrm{N}$ y no presentó diferencias, para los tratamientos fertilizados, entre tipo de fertilizante y momento de aplicación del mismo. El índice de cosecha del N no presentó diferencias significativas para los tratamientos de fertilización. Respecto a los cultivares, no difirieron en la eficiencia de utilización del N, pero sí en su partición. Buck Pronto tuvo mayor índice de cosecha que Buck Charrúa, asociado a su mayor acumulación de $\mathrm{N}$ en grano.

Mientras que la eficiencia agronómica, la eficiencia fisiológica y la fracción aparente recuperada no presentaron diferencias significativas para los tratamientos de fertilización y los sistemas de labranza (Cuadro 4), sí tuvieron diferencias para los cultivares, resultando Buck Pronto más eficiente para todas estas variables, lo que coincide con la tendencia mostrada por dicho cultivar en todos los parámetros anteriormente analizados (Cuadros 1 y 2).

Cuadro 3. Correlaciones, de los valores promedios de todos los tratamientos, entre el $\mathrm{N}$ acumulado en la planta en distintos estados fenológicos y su concentración y acumulación en grano.

\begin{tabular}{lccc}
\hline Variables & $\begin{array}{c}\mathrm{N} \text { grano } \\
(\%)\end{array}$ & $\begin{array}{c}\mathrm{N} \text { grano } \\
(\mathrm{Mg})\end{array}$ & $\begin{array}{c}\mathrm{N} \text { grano } \\
\left(\mathrm{kg} \mathrm{ha}^{-1}\right)\end{array}$ \\
\hline $\mathrm{N}$ antesis & $0,25^{\mathrm{ns}}$ & $0,09^{\text {ns }}$ & $0,42^{* *}$ \\
$\mathrm{~N}$ postantesis & $0,51^{* *}$ & $0,23^{\text {ns }}$ & $0,57^{* *}$ \\
$\mathrm{~N}$ madurez & $0,72^{* *}$ & $0,31^{*}$ & $0,94^{* *}$ \\
$\mathrm{ICN}^{(1)}$ & $0,08^{\text {ns }}$ & $0,46^{* *}$ & $0,39^{* *}$ \\
\hline
\end{tabular}

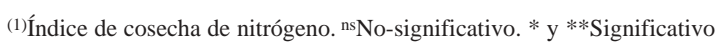
al 0,05 y 0,01 , respectivamente. 
Cuadro 4. Eficiencia de utilización del N (EutN), índice de cosecha del N (ICN), eficiencia agronómica de uso del $\mathrm{N}$ (EA), eficiencia fisiológica (EF), fracción aparente recuperada de N (FAR) y rendimiento (Rendim) en dos cultivares de trigo pan bajo diferentes labranzas y tratamientos de fertilización nitrogenada ${ }^{(1)}$.

\begin{tabular}{|c|c|c|c|c|c|c|}
\hline Tratamiento $^{(2)}$ & $\begin{array}{c}\text { EutN } \\
\left(\mathrm{kg} \mathrm{kg}^{-1}\right)\end{array}$ & $\begin{array}{l}\text { ICN } \\
(\%)\end{array}$ & $\begin{array}{c}\text { EA } \\
\left(\mathrm{kg} \mathrm{kg}^{-1}\right)\end{array}$ & $\begin{array}{c}\mathrm{EF} \\
\left(\mathrm{kg} \mathrm{kg}^{-1}\right)\end{array}$ & $\begin{array}{c}\text { FAR } \\
(\%)\end{array}$ & $\begin{array}{l}\text { Rendim } \\
\left(\mathrm{kg} \mathrm{ha}^{-1}\right)\end{array}$ \\
\hline \multicolumn{7}{|l|}{ Labranza } \\
\hline Reja & $32,0 \mathrm{a}$ & $0,76 \mathrm{a}$ & $12,3 \mathrm{a}$ & $15,8 \mathrm{a}$ & $56,4 \mathrm{a}$ & $621 \mathrm{a}$ \\
\hline Cincel & $28,7 \mathrm{~b}$ & $0,72 b$ & $12,5 \mathrm{a}$ & $21,9 \mathrm{a}$ & $63,2 \mathrm{a}$ & $615 a$ \\
\hline \multicolumn{7}{|l|}{ Fertilización } \\
\hline No & $33,2 \mathrm{a}$ & $0,77 \mathrm{a}$ & - & - & - & $529 \mathrm{~b}$ \\
\hline U90 & $29,8 \mathrm{~b}$ & $0,73 \mathrm{a}$ & $15,4 \mathrm{a}$ & $16,5 \mathrm{a}$ & $79,7 \mathrm{a}$ & $667 a$ \\
\hline Ni90 & $30,2 b$ & $0,75 \mathrm{a}$ & $10,6 \mathrm{a}$ & $20,2 \mathrm{a}$ & $51,6 \mathrm{a}$ & $624 a$ \\
\hline $\mathrm{U} 45+45$ & $28,3 \mathrm{~b}$ & $0,71 \mathrm{a}$ & $10,2 \mathrm{a}$ & $15,6 \mathrm{a}$ & $65,4 \mathrm{a}$ & $621 \mathrm{a}$ \\
\hline $\mathrm{Ni} 45+45$ & $30,3 \mathrm{~b}$ & $0,74 \mathrm{a}$ & $13,4 \mathrm{a}$ & $23,1 \mathrm{a}$ & $60,4 \mathrm{a}$ & $649 \mathrm{a}$ \\
\hline \multicolumn{7}{|l|}{ Cultivares } \\
\hline Buck Pronto & $30,0 \mathrm{a}$ & $0,77 \mathrm{a}$ & $16,3 a$ & $20,8 \mathrm{a}$ & $70,7 \mathrm{a}$ & $678 \mathrm{a}$ \\
\hline Buck Charrúa & $30,7 \mathrm{a}$ & $0,70 \mathrm{~b}$ & $8,4 \mathrm{~b}$ & $16,9 \mathrm{a}$ & $48,8 \mathrm{~b}$ & $558 \mathrm{~b}$ \\
\hline \multicolumn{7}{|c|}{$\begin{array}{l}\text { (1) Letras distintas en la misma columna indican diferencias } \\
\text { estadísticamente significativas entre tratamientos }(\mathrm{P}=0,05) \text { por el test de } \\
\text { Tukey. }{ }^{(2)} \text { Reja: arado de reja y vertedera; Cincel: arado de cinceles; } \\
\text { N0: testigo sin aplicación de } \mathrm{N} \text {; U90: aplicación de } 90 \mathrm{~kg} \mathrm{ha}^{-1} \text { de } \mathrm{N} \text { como } \\
\text { urea a la siembra; Ni90: aplicación de } 90 \mathrm{~kg} \mathrm{ha}^{-1} \mathrm{de} \mathrm{N} \text { como nitrato de } \\
\text { amonio calcáreo a la siembra; } \mathrm{U} 45+45: \mathrm{N} \text { aplicado como urea en dosis de } \\
45 \mathrm{~kg} \mathrm{ha}^{-1} \text { a la siembra más } 45 \mathrm{~kg} \mathrm{ha}^{-1} \text { a fin de macollaje; Ni45+45: } \mathrm{N} \\
\text { aplicado como nitrato de amonio calcáreo en dosis de } 45 \mathrm{~kg} \mathrm{ha}^{-1} \text { a la siembra } \\
\text { más } 45 \mathrm{~kg} \mathrm{ha}^{-1} \text { a fin de macollaje. }\end{array}$} \\
\hline
\end{tabular}

\section{Conclusiones}

1. Existen diferencias en el $\mathrm{N}$ acumulado entre sistemas de labranzas, pero no en el $\mathrm{N}$ contribuido al grano por removilización o por absorción postantesis; el sistema de labranza convencional resulta más eficiente en la utilización y partición del nitrógeno.

2. La fertilización aumenta el $\mathrm{N}$ acumulado y provoca un incremento de la contribución al grano del $\mathrm{N}$ removilizado, pero no mejora el aporte del $\mathrm{N}$ absorbido postantesis, ni aún en la aplicación fraccionada.

3. La eficiencia de utilización del $\mathrm{N}$ disminuye con la aplicación de $\mathrm{N}$, indistintamente para el tipo de fertilizante y momento de aplicación.

4. Los cultivares presentan diferencias en el $\mathrm{N}$ acumulado, en el porcentaje aportado al grano por removilización y absorción postantesis y en la partición del mismo hacia el grano.

\section{Agradecimientos}

A Mirta Castaño por su colaboración en las determinaciones de laboratorio; a la Ing. Agr. María Rosa Simón (MSc.) por las sugerencias brindadas.

\section{Referencias}

ALVAREZ, R.; ALVAREZ, C. R.; DANIEL, P. E.; RICHTER, V.; BLOTTA, L. Nitrogen distribution in soil density fractions and its relation to nitrogen mineralization under tillage systems. Australian Journal of Agricultural Research, Collingwood, v. 36, p. 247-256, 1998.

AUSTIN, R. B.; EDRICH, J. A.; FORD, M. A.; BLACKWELL, R. D. The nitrogen economy of winter wheat. Annual Report of the Plant Breeding Institute, Cambridge, Inglaterra, v. 88, p. 140-141, 1976.

BALBUENA, R. H.; ARAGON, A.; MacDONAGH, P. M.; CLAVERIE, J. A.; TERMINIELLO, A. M. Evolución de la resistencia a la penetración y la densidad aparente en tres sistemas de labranza. In: CONGRESO ARGENTINO DE INGENIERIA RURAL, 4.; CONGRESO INTERNACIONAL DE INGENIERIA RURAL, 2., 1996, Neuquen. Actas... Neuquen: Instituto Nacional de Tecnología Agropecuaria, 1996. p. 197-202.

CALDERINI, D. F.; MIRALLES, D. J.; SLAFER, G. A.; SAVIN, R. Desarrollo, crecimiento y generación del rendimiento en el cultivo de trigo. Buenos Aires: Área de Comunicaciones de Asociación Argentina de Consorcios Regionales de Experimentación Agrícola, 1996. cap. 1, p. 6-17. (Trigo. Cuaderno de Actualización Técnica, 56).

CAMPBELL, C. A.; DAVIDSON, H. R.; McCAIG, T. N. Disposition of nitrogen and soluble sugars in Manitou spring wheat as influenced by $\mathrm{N}$ fertilizer, temperature and duration and stage of moisture stress. Canadian Journal of Plant Science, Ottawa, v. 63, p. 73-90, 1983.

CAMPBELL, C. A.; DE JONG, R. Root-to-strawinfluence of moisture and rate of $\mathrm{N}$ fertilizer. Canadian Journal of Plant Science, Ottawa, v. 81, p. 39-43, 2000.

DALLING, M. J. The physiological basis of nitrogen redistribution during grain filling in cereals. In: HARPER, J. E.; SCHRADER, L. E.; HOWELL, H. W. (Ed.). Exploitation of physiological and genetic variability to enhance crop productivity. Rockville: American Society of Plant Physiology, 1985. p. 55-71.

DELOGU, G.; CATTIVELLI, L.; PECCHIONI, N.; DE FALCIS, D.; MAGGIORE, T.; STANCA, A. M. Uptake and agronomic efficiency of nitrogen in winter barley and winter wheat. European Journal of Agronomy, Amsterdam, v. 9, p. 11-20, 1998.

DORAN, J. W. Soil microbial and biochemical changes associated with reduced tillage. Soil Science Society of America Journal, Madison, v. 44, p. 765-771, 1980. 
FERRERAS, L. A.; BATTISTA, J. J. de; AUSILIO, A.; PECORARI, C. Parámetros físicos del suelo en condiciones no perturbadas y bajo laboreo. Pesquisa Agropecuária Brasileira, Brasília, v. 36, n. 1, p. 161-170, jan. 2001.

GOLIK, S. I.; CHIDICHIMO, H. O. Producción y distribución radical bajo dos sistemas de labranza y nutrición nitrogenada. In: CONGRESO ARGENTINO DE CIENCIA DEL SUELO, 18., 2002, Puerto Madryn. Actas... Puerto Madryn: Asociación Argentina de la Ciencia del Suelo, 2002. 6 p. CD-ROM.

GOLIK, S. I.; CHIDICHIMO, H. O. Trigo: evaluación de la respuesta a distintos sistemas de labranza y alternativas de fertilización. In: CONGRESO NACIONAL DE TRIGO, 5.; SIMPOSIO NACIONAL DE CEREALES DE SIEMBRA OTOÑO INVERNAL, 3., 2002, Villa Carlos Paz. Actas... Villa Carlos Paz: ONENET, 2001. 5 p. CD-ROM.

HEITHOLT, J. J.; CROY, L. I.; MANESS, N. O.; NGUYEN, H. T. Nitrogen partitioning in genotypes of winter wheat differing in grain $\mathrm{N}$ concentration. Field Crops Research, Amsterdam, v. 23, p. 133-144, 1990.

OSAKI, M.; SHINANO, T.; MATSUMOTO, M.; ZHENG, T.; TADANO, T. A root-shoot interaction hypothesis for high productivity of field crops. In: PLANT nutrition for sustainable food production and environment. Tokyo: Kluwer Academic, 1997. p. 669-674.

PALTA, J. A.; FILLERY, R. P. Postanthesis remobilization and losses of nitrogen in wheat in relation to applied nitrogen. Plant and Soil, Dordrecht, v. 155/156, p. 179$181,1993$.

PAPAKOSTA, D. K.; GAGIANAS, A. A. Nitrogen and dry matter accumulation, remobilization, and losses of Mediterranean wheat during grain filling. Agronomy Journal, Madison, v. 83, p. 864-870, 1991

PEREZ, P.; MARTINEZ-CARRASCO, R.; SANCHEZ DE LA PUENTE, L. Uptake and distribution of nitrogen in wheat plants supplied with different amounts of nitrogen after stem elongation. Annals of Applied Biology, Warwick, v. 102, p. 399-406, 1983.

PERUZZO, G.; SIQUEIRA, O. J. F. de; WIETHOLTER, S. Eficiencia agronómica de fertilizantes nitrogenados para a cultura do trigo. Pesquisa Agropecuária Brasileira, Brasília, v. 29, n. 7, p. 1027-1034, jul. 1994.

RUIZ, R. A. Requerimientos de nutrientes y fertilización del cultivo. Buenos Aires: Área de Comunicaciones de Asociación Argentina de Consorcios
Regionales de Experimentación Agrícola, 2001. cap. 3, p. 24-33. (Trigo. Cuaderno de Actualización Técnica, 63).

SARANDÓN, S. J.; CALDIZ, D. O. Effects of varying nitrogen supply at different growth stages on nitrogen uptake and nitrogen partitioning efficiency in two wheat cultivars. Fertilizer Research, Dordrecht, v. 22, p. 2127, 1990 .

SARANDÓN, S. J.; GOLIK, S. I.; CHIDICHIMO, H. O. Acumulación y partición del nitrógeno en dos cultivares de trigo pan ante la fertilización nitrogenada en siembra directa y convencional. Revista de la Facultad de Agronomía, La Plata, v. 102, n. 2, p. 175-186, 1997.

SARQUIS, A.; ALVAREZ, C. R.; ALVAREZ, R. Balance de nitrógeno en trigo bajo distintos manejos. In: CONGRESO ARGENTINO DE CIENCIADEL SUELO, 18., 2002, Puerto Madryn. Actas... Puerto Madryn: Asociación Argentina de la Ciencia del Suelo, 2002. 6 p. CD-ROM.

SPIERTZ, J. H. J.; ELLEN, J. Effects of nitrogen on crop development and grain growth of winter wheat in relation to assimilation and utilization of assimilates and nutrients. Netherlands Journal of Agricultural Science, Amsterdam, v. 26, p. 210-231, 1978.

TABOADA, M. A.; MICUCCI, F. G.; COSENTINO, D. J.; LAVADO, R. S. Comparison of compaction induced by conventional and cero tillage in two soils of the Rolling Pampa of Argentina. Soil and Tillage Research, Amsterdam, v. 49, p. 57-63, 1998.

TAKAHASHI, T.; NAGAO, K.; ITAGAKI, H.; TAKAKU, T. Grain filling mechanisms in spring wheat VI: cultivar variation in nitrogen metabolism and changes in assimilate shortages. Japanese Journal of Crop Science, Tokyo, v. 65, n. 2, p. 289-295, 1996.

TANONI, L. B.; COGLiATTI, D. H. Diferencias varietales de trigo pan en la absorción y partición del nitrógeno y su relación con el contenido de $\mathrm{N}$ en el grano. In: CONGRESO NACIONAL DE TRIGO, 4.; SIMPOSIO NACIONAL DE CULTIVOS DE SIEMBRA OTOÑO INVERNAL, 2., 1998, Mar del Plata, Actas... Mar del Plata: Instituto Nacional de Tecnología Agropecuaria/Universidad Nacional de Mar del Plata, 1998. p. 2-23.

ZADOKS, J. C.; CHANG, T. T.; KONZAK, C. F. A decimal code for the growth stages of cereals. Weed Research, Oxford, v. 14, p. 415- 421, 1974. 